'Unidad de Medicina Reproductiva, Clínica Las Condes. Santiago, Chile.

2Programa de Ética y Políticas Públicas en Reproducción Humana.

${ }^{3}$ Facultad de Medicina Universidad Diego Portales. Santiago, Chile. aph.D.

Manuscrito por invitación de los editores.

Este trabajo no tuvo apoyo financiero.

Recibido el 2 de junio de 2014, aceptado el 20 de junio de 2014

Correspondencia a:

Dr. Fernando Zegers-Hochschild Ejército 260, Santiago, Chile. fzegers@clc.cl

\section{Fundamentos biomédicos y éticos de la criopreservación de embriones}

\author{
FERNANDO ZEGERS-HOCHSCHILD ${ }^{1,2}$, \\ JAVIER A. CROSBY ${ }^{1, a}$, SOFÍA P. SALAS ${ }^{2,3}$
}

\section{Medical and ethical basis for embryo cryopreservation}

\begin{abstract}
As part of Assisted Reproductive Technologies (ART), the advent of embryo freezing lowered the number of embryos transferred, decreasing multiple births without jeopardizing pregnancy rates. Using vitrification technology, 90\% of embryos survive after thawing, producing clinical pregnancy rates similar to those of fresh embryos (41.6\% y $44.3 \%$ respectively). Furthermore, cumulative pregnancy rates, obtained after transferring fresh plus frozen/thawed embryos, can reach $70 \%$. Frozen embryo transfers (FET) are reported by six of seven institutions, which are part of the Chilean ART registry, and altogether constitute $22.8 \%$ of all ART procedures. Increasing use of cryopreservation lowered overall multiple gestations from 33\% in 1995 to 23\% in 2011, reducing pre term births and perinatal mortality. For many people, embryo freezing generates ethical dilemmas, due to the potential risks to which embryos are exposed, and the uncontrolled accumulation and disposal of human embryos. Scientific evidence today shows that frozen/thawed embryos are not exposed to disproportionate risks, and by hindering its use, both women and their children are exposed to the risks of multiple gestation, repeated cycles of ovarian hormonal stimulation or the impossibility to afford repeated ART cycles. In this article, we provide biomedical, as well as ethical, arguments to sustain that embryo cryopreservation is not only justified but fundamental when offering infertility treatment with ART.
\end{abstract}

(Rev Med Chile 2014; 142: 896-902)

Key words: Cryopreservation; Embryo dispositions; Embryo research; Legislation; Moral obligations; Reproductive techniques.
$\mathrm{D}$ esde el reporte en 1983 del primer embarazo obtenido mediante transferencia de embriones fecundados in vitro y criopreservados ${ }^{1}$, la biotecnología mejoró los medios crio-protectores, sistemas de almacenamiento y protocolos de congelación, haciendo posible una adecuada sobrevida embrionaria al descongelar y tasas de embarazos semejantes a la transferencia de embriones frescos (no congelados). El gran cambio se logró hace una década, con el desarrollo de un sistema ultra rápido de vitrificación ${ }^{2}$, que evita la formación de hielo, permitiendo el paso de una fase líquida a una sólida en fracción de segundos, mejorando la estabilidad y el mantenimiento del esqueleto celular. Estos avances lograron tasas de sobrevida embrionaria al descongelar entre $90 \mathrm{y}$ $97 \%$, cuando los embriones se criopreservan en estado de blastocistos, es decir, al quinto día del desarrollo in vitro ${ }^{3}$. Actualmente, la vitrificación de blastocistos es aceptada por la Sociedad Americana de Medicina Reproductiva y la Sociedad Europea de Reproducción Humana y Embriología como un tratamiento médico seguro y eficaz 4 .

Para comprender mejor la necesidad de usar la criopreservación embrionaria en tratamientos de TRA, es necesario conocer algunos fundamentos biológicos del proceso reproductivo humano. 
1. La probabilidad de embarazo en mujeres sanas $<35$ años, luego de relaciones sexuales en el período fértil, es sólo $25 \%$ a $26 \%$ y la tasa acumulativa de embarazo luego de tres ciclos no supera el $50 \%$ de las mujeres ${ }^{5}$.

2. En mujeres $<35$ años, la probabilidad de embarazo al transferir un embrión producto de una FIV es de $25 \%$ a $35 \%$, mientras que la probabilidad de embarazo al transferir dos embriones sube a $45 \%-50 \%$, con una tasa de gemelos del orden de $26 \%$. Al transferir tres embriones, la tasa de embarazo no aumenta significativamente, pero se originan embarazos múltiples (triples y cuádruples) en $1,6 \%$ a 3,5\% de los casos ${ }^{6}$.

3.- La muerte embrionaria es parte del proceso natural y ocurre de manera semejante en el oviducto humano y en los medios de cultivo extra corpóreos. La Figura 1 muestra la dinámica del desarrollo y muerte embrionaria pre-implantacional en más de 500 embriones cultivados in vitro en el Programa de Reproducción Asistida de Clínica Las Condes. El 11\% de los embriones mueren antes del segundo día; $23 \%$ entre el segundo y tercer día, y no más de la mitad logran llegar a blastocisto, etapa previa a la implantación. Es decir, en nuestra especie, tan sólo $47 \%$ de los óvulos fecundados son susceptibles de tomar contacto con el endometrio y eventualmente implantarse.

4. Es interesante señalar que gran parte de estas muertes embrionarias son el resultado de errores cromosómicos secundarios a una alta frecuencia de aneuploidía ovocitaria. La Figura 2 , muestra la frecuencia de aneuploidía en polocitos obtenidos de 945 ovocitos de mujeres en diferentes edades, mediante tecnología de fluorescencia (FISH) aplicada en los cromosomas $13,16,18,21, y 22$. La frecuencia de aneuploidía fluctúa entre $21 \%$ en mujeres de 24 a 34 años, hasta $97 \%$ en mujeres de 44 a 47 años ${ }^{7}$.

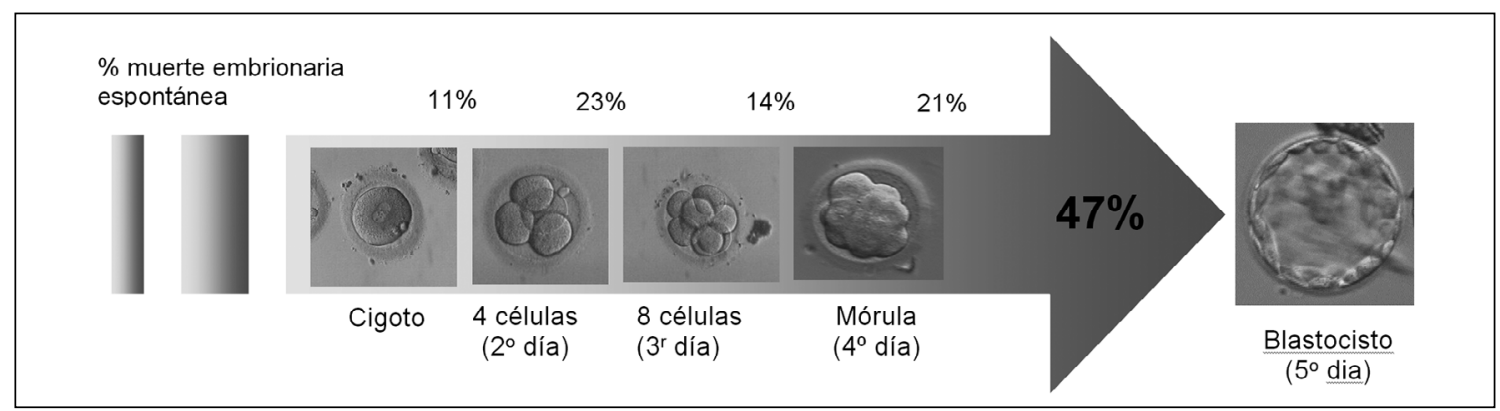

Figura 1. Dinámica del desarrollo embrionario pre-implantación analizado en 572 cigotos de 74 mujeres transferidas en día $5^{\circ}$ de desarrollo. Unidad de Medicina Reproductiva, Clínica Las Condes.

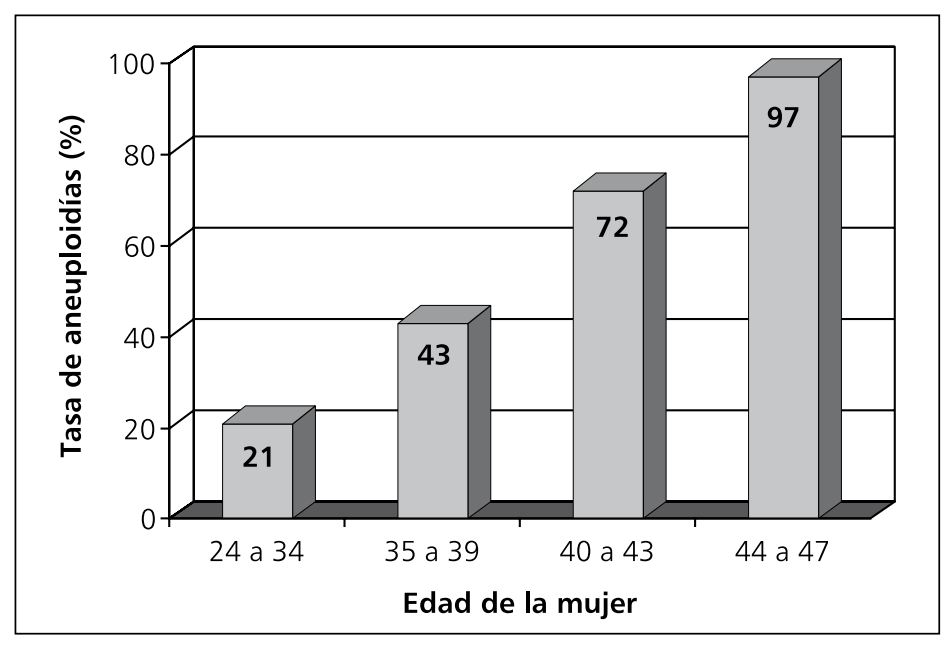

Figura 2. Frecuencia de aneuploidías para 5 cromosomas $(13,16,18,21,22)$ analizada en 945 polocitos humanos de acuerdo a la edad de la mujer. Unidad de Medicina Reproductiva, Clínica Las Condes. 
Dada esta realidad biológica, es necesario preguntarse cómo procurar un tratamiento de reproducción asistida seguro y eficiente, considerando la falta de predictibilidad individual respecto de cuántos óvulos lograrán ser fecundados, y cuántos de los cigotos llegarán a blastocisto y tendrán la capacidad de implantarse en el endometrio.

La comunidad médica respondió a este dilema con la criopreservación embrionaria, que permite, entre otras cosas: a) estimular hormonalmente a la mujer con el objeto de obtener varios óvulos; b) fecundar los óvulos maduros; c) esperar a que ocurran las muertes embrionarias que deben ocurrir y si uno o más embriones llegan al estado de blastocisto, transferir a la mujer uno o máximo dos embriones, y d) si hay embriones remanentes, vitrificarlos para una futura transferencia.

\section{Realidad de la criopreservación embrionaria en Chile y el mundo}

Anualmente se registran entre 1 y 1,3 millones de ciclos de TRA y nacen 237.300 a 300.000 bebés. El último reporte mundial indica que $28 \%$ de los ciclos de reproducción autóloga corresponden a la transferencia de embriones congelados/descongelados (TEC). El aumento en la proporción de TEC se asocia a una disminución proporcional de partos múltiples, sobretodo multiparidad de alto orden $^{8}$. La misma situación se observa en Latino América, donde la proporción de TEC aumentó de $7 \%$ a 24,6\% entre los años 1995 y $2011^{9,10}$. En Chile, entre 1997 y 2009, la TEC aumentó de $10 \%$ a $22,8 \%{ }^{11}$. El reporte de 6 de 7 centros nacionales (www.redlara.com) incluye 325 ciclos de TEC de un total de 1,427 procedimientos de reproducción autóloga $(22,8 \%)$.

$\mathrm{Al}$ igual que en el resto del mundo, en Chile existe un paralelo entre el aumento en la proporción de embarazos generados con TEC y una disminución en la tasa de multigestación; entre 1995 y 2009, la proporción de embarazo gemelar bajó de $33 \%$ a $20 \%$ y los embarazos de alto orden disminuyeron de $15 \%$ a $2 \%$.

\section{¿Por qué es importante la prevención de multigestación?}

La multigestación, sobretodo la extrema, constituye la iatrogenia más frecuente en las TRA. Las complicaciones para los bebés nacidos de embarazos múltiples ocurren de manera independiente de si éstos se generaron de manera espontánea o mediante reproducción médicamente asistida, y derivan fundamentalmente de prematurez. La Tabla 1 muestra que la mortalidad perinatal aumenta de 10 por mil en partos únicos a 28 por mil en gemelos, y 64 y 187 por mil en triples y cuádruples, respectivamente. Por otra parte, la morbilidad neonatal e infantil también aumenta como consecuencia de la prematurez. Desde una perspectiva social, es necesario recordar que si bien la llegada de más de un hijo a familias infértiles es casi siempre bien recibida, esto puede afectar seriamente la estabilidad económica, laboral y social de la pareja. Los severos riesgos para las mujeres y para los bebés que nacen de partos múltiples, ha hecho que en los países que tienen regulaciones estatales para el ejercicio de TRA, principalmente en Europa, Asia y Oceanía, se restrinja la transferencia a sólo uno o máximo dos embriones.

Tabla 1. Mortalidad Perinatal de acuerdo al orden de gestación

\begin{tabular}{|c|c|c|c|c|}
\hline & Único & Doble & Triple & $\geq$ Cuádruple \\
\hline Bebés nacidos & 50.501 & 31.814 & 9.318 & 1.158 \\
\hline Nacidos vivos & 50.191 & 31.270 & 9.017 & 1.071 \\
\hline Mortinatos & $310(6 \%)$ & $544(17 \%$ o $)$ & 301 (32\%o) & $87(75 \% \circ)$ \\
\hline Mortineonatos & $190(4 \%)$ & $339(11 \%$ o) & 294 (32\%o) & $130(112 \%)$ \\
\hline Mortalidad perinatal & $10 \%$ & $28 \%$ & $64 \%$ & $187 \%$ \\
\hline
\end{tabular}

Registro Latino Americano, 1990-20099. 
Resultados clínicos de la vitrificación embrionaria en el Programa de Reproducción Asistida en Clínica Las Condes

Los resultados clínicos de programas de criopreservación embrionaria varían de acuerdo a políticas institucionales, tecnologías usadas, decisiones respecto de cuales embriones criopreservar y en qué estadio de desarrollo embrionario. El desarrollo de nuevos métodos de criopreservación, permitió demostrar que cuando los embriones se criopreservan en el día $5^{\circ}$ en vez del día $3^{\circ}$ de desarrollo embrionario, la sobrevida post congelación aumenta significativamente, por lo que en la actualidad la mayoría de los centros realiza el procedimiento en estado de blastocisto, permitiendo que la muerte natural de embriones (que de todas maneras sucede como parte del proceso reproductivo humano), ocurra espontáneamente. Así, sólo se criopreservan los embriones que han sobrepasado esa barrera natural. En Latino América, el último reporte global ${ }^{10}$ demuestra que no hay diferencias significativas en las tasas de embarazo y parto al transferir embriones frescos y congelados. En el año 2011, de un total de 26.321 procedimientos con embriones frescos en Latino América, las tasas de embarazo y parto fueron $29,8 \%$ y $20,7 \%$, respectivamente. En ese mismo período, de 6.909 transferencias de embriones criopreservados, las tasas de embarazo y parto fueron $35,2 \%$ y $24,3 \%$, respectivamente. En Clínica Las Condes, entre 2011 y 2013 se realizaron 1.227 ciclos de TRA con embriones frescos y 322 ciclos de descongelación de embriones vitrificados. Como se aprecia en la Tabla 2, que corresponde a información científica publicada por primera vez, la sobrevida embrionaria luego de la descongelación fue de $89,9 \%$ y, a pesar que la media de embriones transferidos es menor en el grupo de TEC, la tasa de embarazo y aborto es en todo similar a la obtenida al transferir embriones frescos. Es decir, la vitrificación embrionaria no afecta negativamente la posibilidad de embarazo y no genera un número mayor de abortos. Al sumar los embarazos luego de transferir los embriones frescos y los criopreservados de cada persona, la tasa acumulativa de embarazo en mujeres de 30 a 40 años llega a $72 \%$ (Tabla 3). Es importante considerar que todos estos casos corresponden a vitrificación de blastocistos. Es decir, esta tasa acumulada de embarazo corresponde a un grupo seleccionado de mujeres más eficientes pues tuvieron uno o dos embriones para transferir en fresco, además de embriones supernumerarios, vitrificados en etapa de blastocisto.

Es razonable concluir que la vitrificación de blastocistos no afecta la posibilidad de embarazo y permite duplicar las tasas de embarazo que se logran al transferir un máximo de dos embriones en un ciclo fresco.

En Clínica Las Condes, la mujer y su pareja deben firmar un formulario de consentimiento, mediante el cual aceptan la vitrificación de em-

Tabla 2. Resultados generales de procedimientos de TRA con embriones frescos y desvitrificados

\begin{tabular}{|lcc|}
\hline $\begin{array}{l}\text { Transferencia } \\
\text { de embriones }\end{array}$ & Frescos & $\begin{array}{c}\text { Desvitri- } \\
\text { ficados }\end{array}$ \\
$\begin{array}{l}\text { Ciclos aspirados/Ciclos } \\
\text { desvitrificados }\end{array}$ & 1.227 & 322 \\
$\begin{array}{l}\text { Ciclos transferidos } \\
\text { Mediana de la edad }\end{array}$ & 987 & 293 \\
$\begin{array}{l}\text { Tasa de fecundación/Sobrevida } \\
\text { al desvitrificar }\end{array}$ & $72,1 \%$ & 35 \\
$\begin{array}{l}\text { Media de embriones transferidos } \\
\text { Embarazos clínicos }\end{array}$ & 2,03 & 1,76 \\
$\begin{array}{l}\text { Tasa de embarazo por } \\
\text { transferencia }\end{array}$ & 437 & 122 \\
\hline $\begin{array}{l}\text { Tasa de aborto } \\
\text { M }\end{array}$ & $44,3 \%$ & $41,6 \% *$ \\
\hline
\end{tabular}

${ }^{*} p=0,4568 .{ }^{* *} p=0,7218$. Unidad de Medicina Reproductiva, Clínica Las Condes.

Tabla 3. Tasa acumulativa de embarazo luego de la transferencia de embriones frescos y vitrificados

\begin{tabular}{|lc|}
\hline Día de transferencia/vitrificación & $\mathbf{3}^{\text {er }} \mathbf{5}^{\text {to }}$ día \\
\hline Transferencias en fresco & 222 \\
Media de la edad materna & 34.6 \\
\hline Embarazos en fresco & $117(52,7 \%)$ \\
\hline Transferencias de embriones vitrificados & 104 \\
\hline Embarazos con embriones vitrificados & $43(41,3 \%)$ \\
\hline Embarazos totales & 160 \\
\hline Tasa acumulativa de embarazo & $72,1 \%$ \\
Abortos & $18(11,3 \%)$ \\
\hline
\end{tabular}

Unidad de Medicina Reproductiva, Clínica Las Condes, 20092013. 
Tabla 4. Decisiones respecto de vitrificar o no blastocistos en la Unidad de Medicina Reproductiva, Clínica Las Condes, 2011-2013

\begin{tabular}{|lrr|}
\hline Ciclos de tratamiento & 1.117 & \\
\hline Decisión de NO vitrificar & 43 & $3,8 \%$ \\
\hline Intención de vitrificar sólo ovocitos & 9 & $0,8 \%$ \\
\hline Intención de vitrificar blastocistos & 1.038 & $95,4 \%$ \\
Pacientes que vitrificaron embriones & 330 & $31,8 \%$ \\
$1-2$ & 232 & $70,3 \%$ \\
$3-5$ & 87 & $26,4 \%$ \\
$>5$ & 11 & $3,3 \%$ \\
\hline
\end{tabular}

Unidad de Medicina Reproductiva, Clínica Las Condes, 2011 2013.

briones; en éste se explicita que los embriones criopreservados no pueden ser eliminados. Esto significa que las parejas acceden a que si luego del plazo establecido, ella no desea que sus embriones le sean transferidos, debe donarlos a terceros, sin obtener compensación alguna. En la Tabla 4 se aprecia que de un total de 1.117 mujeres que realizaron TRA, sólo 4,6\% expresó que no quería vitrificar o que sólo vitrificaba óvulos. Así, 95,4\% de las mujeres y sus parejas que se realizan procedimientos de TRA en Clínica Las Condes, quieren vitrificar embriones para armonizar adecuadas tasas de embarazo, evitando la multigestación extrema. En todo caso, es interesante considerar que en un programa de criopreservación de blastocistos, sólo un tercio de las personas que manifestaron su voluntad a criopreservar logra hacerlo.

Así, la evidencia científica muestra que la vitrificación en estado de blastocisto no parece afectar la posibilidad del embrión de iniciar una gestación y permite equilibrar una adecuada chance de embarazo acumulativo, minimizando los riesgos inherentes a la multigestación extrema y a la estimulación ovárica repetida, con evidentes beneficios para la mujer, los bebés y la familia.

\section{Algunas consideraciones bioéticas y de políticas públicas}

Muchos estudios psicosociales demuestran que para las mujeres y sus parejas, criopreservar embriones no es inocuo. Aún en países donde es permitido desechar embriones, mantener embriones vitrificados genera conflictos en sus progenitores $^{12}$. En Chile, la ausencia de un ente regulador hace que diferentes instituciones ofrezcan distintas estrategias en relación al devenir de los embriones criopreservados. Así por ejemplo, en Clínica Las Condes no está permitido desechar embriones, lo que obliga a las parejas que no quieren tener más hijos, a donarlos a terceros. La sola idea de tener que donar embriones atemoriza a muchas parejas. Por ello, a pesar de las dificultades para predecir el devenir de la fecundación in vitro, es buena práctica médica limitar el número de óvulos a inseminar a aquellos que son capaces de generar un número de embriones que permita una transferencia en fresco e idealmente sólo una transferencia más con embriones vitrificados. De esa manera, las parejas realizan el procedimiento con una posibilidad de alrededor de 70\% de lograr un embarazo clínico, sin tener que hacerse cargo de un exceso de embriones.

Como ya se dijo, la generación de partos múltiples constituye la más seria complicación de las TRA. Desde la perspectiva del deber ético para con las personas y sus hijos, la criopreservación embrionaria es la única manera de lograr un adecuado balance que busca mantener un relativo éxito terapéutico, minimizando el riesgo de complicaciones neonatales y maternas derivadas de la multigestación. En general, no es aconsejable y para algunos, no es ético transferir más de dos embriones, sobre todo en mujeres jóvenes. De este concepto se derivan al menos tres reflexiones:

\section{a) ¿Es ético realizar procedimientos de fecundación asistida sin criopreservación embrionaria? \\ Nuestro pensamiento es que es ético, en la} medida que la pareja que solicita el tratamiento entiende exactamente las limitantes y las bajas posibilidades de éxito en esas condiciones. Más aún, no sería ético negarles el tratamiento a parejas por el hecho de que la alternativa terapéutica elegida por ellos sea menos exitosa. Esa decisión debe ser de la pareja y no del médico. Lo que no es ético, en esas circunstancias, es transferir más embriones para aumentar las posibilidades de éxito y compensar la falta de criopreservación. Aunque la mujer exprese su voluntad de exponerse al riesgo de una gestación triple, ella no tiene derecho a someter a sus hijos/as a ese riesgo. 
b) ¿Es ético someter a una pareja a los riesgos y costos económicos de una fecundación asistida y negarles la oportunidad de criopreservar embriones?

Es razonable argumentar que no debe forzarse a una pareja a tratamientos costosos y de riesgo, privándolos de la autonomía para hacer uso de los recursos médicamente valorados como seguros y eficientes para prevenir la multigestación, manteniendo tasas aceptables de embarazo. La criopreservación embrionaria debe ser ofrecida como alternativa terapéutica, sobre todo en medios donde el costo del tratamiento es financiado por la propia pareja. No es posible establecer un programa de transferencia de un solo embrión evitando la multigestación, si no se criopreservan embriones.

c) ¿Es ético obligar a una pareja a transferir sólo un embrión (criopreservando el resto) para prevenir la multigestación?

Esta política, que es usada en varios países de Europa, es éticamente aceptable y deseable en la medida que el mismo estado que obliga, garantice el acceso gratuito a los tratamientos, incluyendo el embarazo y parto. La razón de ello es que si el estado financia estas prestaciones de salud con los impuestos de todos, también puede poner condiciones que disminuyan los riesgos y los costos neonatales derivados de dichos tratamientos, en la medida que ello no someta a las parejas a tratamientos menos exitosos o a riesgos para su salud.

La criopreservación embrionaria se realiza en todo el mundo, con excepción de Alemania e Irlanda. En la mayor parte de los países nórdicos, Japón y Australia, se practica la transferencia electiva de sólo un embrión en fresco, seguido de la transferencia de embriones congelados/ descongelados. Con ello, se ha minimizado el embarazo gemelar a niveles inferiores a 5\%, manteniendo una tasa acumulada de embarazo igual a la obtenida con transferencia en fresco de 2 y 3 embriones, que reportan tasas de multigestación que superan el $30 \%$.

Los derechos humanos universales le confieren a las personas el beneficio a acceder a los avances de la ciencia para satisfacer sus aspiraciones y recibir cuidados médicos seguros y eficientes. Es en este contexto que la criopreservación embrionaria forma parte del arsenal terapéutico que no puede faltar en un programa de reproducción asistida.

\section{El derecho a la autonomía}

Como parte del respeto a la autonomía de las parejas que voluntariamente se someten a procedimientos de TRA para fundar una familia, es necesario procurar que las personas puedan elegir los caminos terapéuticos que sean armónicos con sus creencias y valores. Las alternativas para aquellas parejas que no están dispuestas a criopreservar embriones, es hacer TRA con un máximo de dos óvulos expuestos a fecundación (número máximo de embriones a transferir) y en lo posible criopreservar ovocitos. La experiencia personal demuestra que si se quiere evitar la multigestación extrema, las tasas de parto sin criopreservación de embriones no superan $20 \%$ por ciclo. Entonces, en el $80 \%$ de los casos será necesario repetir un ciclo completo si la pareja tiene los recursos económicos y la salud general para hacerlo. Es importante señalar que mujeres con insuficiencia ovárica prematura y hombres con falla testicular, tienen habitualmente sólo una oportunidad para generar los embriones con los que formarán su familia.

La criopreservación de ovocitos ha experimentado enormes avances, pero es una tecnología complementaria a la criopreservación embrionaria. Esto es así porque al momento de querer usar esos óvulos vitrificados, el dilema de cuantos óvulos desvitrificar y cuántos de ellos intentar fecundar es aún más complejo que con óvulos frescos. La mayor complejidad radica en que la predictibilidad de la tasa de fecundación y desarrollo embrionario in vitro, es aun más incierta con óvulos desvitrificados que con óvulos frescos; y por ello siempre se hace necesario tener la alternativa de vitrificar embriones, aun como medida de "salvataje". Hoy, la vitrificación de óvulos es una gran alternativa terapéutica en donación de óvulos y para preservar la fertilidad en mujeres que serán sometidas a radioterapia, quimioterapia o cirugía ovárica. También es una gran alternativa para mujeres que desean postergar su fertilidad por razones sociales personales.

\section{Destino de embriones criopreservados}

Otro aspecto que genera importante discusión ética se refiere al destino de los embriones criopreservados, especialmente si hay desacuerdos en la pareja. Es aconsejable que en el consentimiento 
informado previo al procedimiento de TRA, se establezcan los posibles cursos de acción cuando existan desavenencias entre los progenitores. En términos generales, nos parece que antes de recurrir a la donación de embriones, es mejor que estos sean transferidos a su progenitora, si así lo solicita, o pueda encontrar una receptora ligada a su progenitor.

\section{Efecto de la criopreservación embrionaria en la salud de los niños nacidos}

Múltiples estudios examinan el peso de los recién nacidos y las tasas de malformaciones en bebés generados mediante TRA y criopreservación embrionaria.

Una reciente revisión ${ }^{13}$, demostró que el peso al nacer de los bebés concebidos mediante criopreservación y vitrificación, supera el peso de los nacidos de embriones frescos y no se diferencia del peso de los nacidos mediante reproducción espontánea. Por otra parte, la tasa de malformaciones $(1,4 \%)$ en más de 900 nacidos de criopreservación embrionaria fue similar a los nacidos mediante reproducción espontánea. En Latino América, la tasa de malformaciones en 1.330 nacidos de TEC fue $1,4 \%$ (igual a los países nórdicos) comparado con 1,9\% en 16.449 nacidos de embriones frescos ${ }^{9}$. En general, se piensa que la criopreservación protege a los embriones contra algunas alteraciones del desarrollo, pero no en lo que respecta a malformaciones generales. Si bien los datos en la actualidad son tranquilizantes, es necesario mantener una vigilancia estricta sobre el devenir de estos niños, ya que la proporción de nacidos de embriones criopreservados será cada vez mayor en el mundo, en la medida que se intenta restringir el nacimiento de bebés que resultan de partos múltiples.

\section{Referencias}

1. Trounson A, Mohr L. Human pregnancy following cryopreservation, thawing and transfer of an eight-cell embryo. Nature 1983; 305: 707-9.

2. Kuwayama M, Vajta G, Kato O, Leibo SP. Highly efficient vitrification method for cryopreservation of human oocytes. RBM Online 2005; 11 (3): 300-8.

3. Cobo A, Kuwayama M, Pérez S, Ruiz A, Pellicer A, Remohi J. Comparison of concomitant outcome achieved with fresh and cryopreserved donor oocytes vitrified by the Cryotop method. Fertil Steril 2008; 89 (6): 1657-64.

4. Gianaroli L, Racowsky C, Geraedts J, Cedars M, Makrigiannakis A, Lobo R. Best practices of ASRM and ESHRE: a journey through reproductive medicine. Hum Reprod 2012; 27 (12): 3365-79.

5. te Velde ER, Eijkemans R, Habbema HDF. Variation in couple fecundity and time to pregnancy, an essential concept in human reproduction. The Lancet 2000; 355 : 1928-9.

6. Zegers-Hochschild F, Schwarze JE, Crosby JA, Musri C, Borges de Souza MC. Assisted Reproductive Ttechnologies (ART) in Latin America: The Latin American Registry, 2011. JBRA Assit Reprod 2013; 17 (4): 216-23.

7. Fabres C, Fernández E, Zegers-Hochschild F, Mackenna A, Crosby JA. First polar body genetic diagnosis (PbGD) as a selection tool of euploid oocytes before insemination. JBRA Assist Reprod 2012; 16 (4): 231-4.

8. Zegers-Hochschild F, Mansour R, Ishihara O, Adamson GD, de Mouzon J, Nygren KG, et al. International Committee for Monitoring Assisted Reproductive Technology: world report on assisted reproductive technology, 2005. Fertil Steril 2013; 101 (2): 366-78.

9. Zegers-Hochschild F, Schwarze JE, Crosby JA, Borges de Souza MC. Twenty years of Assisted Reproductive Technology (ART) in Latin America. JBRA Assit Reprod 2011; 15 (2): 19-30.

10. Schwarze JE, Crosby JA, Vantman D, Masoli P, Ciuffardi I, Céspedes P, et al. Registro Chileno de Técnicas de Reproducción Asistida Realizadas en el 2011. Rev Chil Obstet Ginecol 2014. Aceptado para publicación.

11. Schwarze JE, Crosby JA, Zegers-Hochschild F. 20 años de Reproducción Asistida en Chile 1990-2009. Publicado en la página web de la Sociedad Chilena de Medicina Reproductiva: http://www.socmer.org/registro-chileno-1990-2009.pdf

12. Nachtigall RD, Mac Dougall K, Harrington J, Duff J, Becker G. How couples who have undergone in vitro fertilization decide what to do with surplus frozen embryos. Fertil Steril 2009; 92 (6): 2094-6.

13. Wennerholm UB, Henningsen AK, Romundstad LB, Bergh C, Pinborg A, Skjaerven R, et al. Perinatal outcomes of children born after frozen-thawed embryo transfer: a Nordic cohort study from the CoNARTaS group. Hum Reprod 2013; 28 (9): 2545-53. 